\title{
The Value of UK Respiratory Conditions Research on Clinical Practice Guidelines from Other European Member States
}

\section{Elena Pallari ${ }^{1,2 *}$}

${ }^{1}$ King's College London, Institute of Psychiatry, Psychology \& Neuroscience, Centre for Implementation Science, De Crespigny Park, Denmark Hill, London, SE5 8AF, United Kingdom

${ }^{2}$ King's College London, Institute of Pharmaceutical Sciences, Academic Centre, Franklin-Wilkins Building, King's College London, 150 Stamford Street, London, SE1 9NH, United Kingdom

\begin{abstract}
Background/objectives: Research on chronic non-communicable respiratory conditions is limited. This study assessed the impact of UK medical research on other European countries development of clinical practice guidelines (CPGs), to evaluate the UK contribution to the evidence-base of European CPGs on chronic non-communicable respiratory conditions.
\end{abstract}

Method: The UK contribution was determined on a fractional count basis to clinical guidelines' citations: (1) from each of five selected (target) Member States (MS), namely France, Germany, The Netherlands, Sweden and Switzerland; and (2) from the other 25 countries (the other 23 EU MS, plus Iceland and Norway).

Results: There were 6,087 cited references on 52 clinical practice guidelines published from 21 European countries. The UK contribution varied between $11 \%$ and $17 \%$ for the five target EU MSs countries for all respiratory conditions clinical guidelines. There was more foreign collaboration research on Chronic Obstructive Pulmonary Disease (COPD) $(33 \%)$ than asthma $(25 \%)$ with results being statistically significant.

Conclusions: The study showed the importance of UK medical research contribution to the evidence-base of respiratory clinical guidelines in other European MS. Notable differences between asthma and COPD, can inform research priorities and medical progression on respiratory conditions' clinical management.

Keywords: Respiratory conditions; Clinical practice guidelines; United Kingdom; European Union; Research impact

\section{Introduction}

Clinical practice guidelines (CPGs) are being increasingly used to provide recommendations based on the best available evidence to assist clinicians in effective decision-making towards the care of the patient as well as decrease variation of care [1-3]. Studies demonstrated that their use does change clinical practice and improve the quality of patient care, despite variations of reported outcomes [4-6].

Research on chronic non-communicable respiratory conditions is limited with only $0.77 \%$ of the overall biomedical research output in Europe attributed to these and $0.55 \%$ in the world between 2002 and 2013, while their disease burden accounts eight-times more $[7,8]$. Additionally, research in this field is unresearched with a $2.5 \%-4.5 \%$ funding across biomedical research and $4.3 \%$ within the European Union's (EU) seventh Framework Programme for Research and Technological Development (FP7) [8,9]. For the period 2007-2013 the UK received a total of $€ 8.8$ billion from EU, while the EU received $€ 77.7$ billion worth of UK funding [10].

Throughout Europe there are different organisations involved in the development of CPGs for a range of diseases, acute or chronic conditions. As part of a European Commission research project on the evaluation of the impact of non-communicable diseases, this short piece of research work focuses on the assessment of the extent of UK influence on the cited research evidence-base of CPGs development in other European countries. The set objectives were to identify CPGs on chronic respiratory conditions, collect their underpinning cited references and analyse the contribution of UK research in the form of evidence-base references from these guidelines. This was done for those respiratory CPGs causing the most disease burden on the average European population (see methods section).

\section{Methods}

\section{Selection of clinical practice guidelines}

For this work, the cited references underpinning the clinical care recommendations within CPGs were analysed across Europe. The clinical guidelines were identified through the websites of each development body within each European country. In France, there is an independent public authority responsible for the quality of patient care and the publication of clinical practice guidelines, the Haute Authorite de Santé, HAS (the French National Authority for Health) [11,12]. In Germany the process of CPGs development is centralised from the Working Group of the Scientific Medical Societies (Arbeitsgemeinschaft der Wissenschaftlichen Medizinischen Fachgesellschaften (AWMF)) $[11,13]$ which is the umbrella organization of 158 medical societies [10], and the Agency for Quality in Medicine (ÄZQ) through the National Disease Management Guideline Programme (Nationale VersorgungsLeitlinien (NVL)) [14]. In Netherlands there are various organisations involved in the development of CPGs like the National Institute for Public Health and the Environment (RIVM), the Dutch Institute for Healthcare Improvement (CBO), the Dutch Council for Quality of Care and the Dutch College of General Practitioners

*Corresponding author: Elena Pallari, King's College London, Institute of Psychiatry, Psychology \& Neuroscience, Centre for Implementation Science, De Crespigny Park, Denmark Hill, London, SE5 8AF, United Kingdom, Tel: 02078480683; E-mail: elena.pallari@kcl.ac.uk

Received November 24, 2017; Accepted December 04, 2017; Published December 11, 2017

Citation: Pallari E (2017) The Value of UK Respiratory Conditions Research on Clinical Practice Guidelines from Other European Member States . J Pulm Respir Med 7: 434. doi: 10.4172/2161-105X.1000434

Copyright: (C) 2017 Pallari E. This is an open-access article distributed under the terms of the Creative Commons Attribution License, which permits unrestricted use, distribution, and reproduction in any medium, provided the original author and source are credited. 
(NHG) involved with all guidelines available through one electronic platform [15]. The National Board of Health and Welfare (NBHW) under the Ministry of Health and Social Affairs (Socialstyrelsen) in Sweden is responsible for the development of guidelines at the national level $[11,16]$. In Switzerland the process of CPGs development is decentralised with various organisations involved, and with the Swiss Respiratory Society overseeing the only CPG identified on COPD [17].

For a list of the involved organisations and identified clinical guidelines, see Table 1 .

KCL graduate students were recruited and trained to identify clinical guidelines in the field of respiratory conditions for the 28 European Union Member States and three European Free Trade Association countries: Iceland, Switzerland and Norway (EUR31). The study period was 2002-2013 for the publication of the clinical guidelines; however, some of the cited references were published well before 2002, as 2013 accepted articles were published in 2014. EP then identified the cited references, downloaded their details from the Web of Science ${ }^{\bullet}$ Clarivate Analytics and using VBA programs their publication details were extracted on an Excel spreadsheet. Due to the high volume of clinical guidelines published in the EUR31 countries during 2002-2013, the work was limited to an analysis of those that dealt with diseases and disorders causing $1 \%$ or more of the European disease burden, as measured by Disability-Adjusted Life Years (DALYs) [2]. For respiratory conditions DALYs affecting the European population $2.95 \%$ are due to COPD and $1.07 \%$ due to asthma, shown in Table 2.

\section{Data processing}

The details of the cited references within the clinical guidelines were prepared as a series of search statements on Excel, including keywords from the title, publication year and author and run against the Web of Science (WoS). Their full bibliographic details were downloaded as text files from the WoS and were then converted by means of a VBA program into an Excel spreadsheet. An additional VBA program was used for an analysis of the addresses on each paper, and the fractional count of each country was tabulated in a separate column of the spreadsheet. The titles of the papers were matched to those of cited papers in the respiratory clinical guidelines and other details including address, publication year, journal and funding information was recorded.

\section{Data analysis}

The main analyses performed was of the contribution of the UK to cited papers in the respiratory clinical guidelines in the following way: (A) from each of the five selected (target) MS, namely, France (FR), Germany (DE), The Netherlands (NL), Sweden (SE) and Switzerland $(\mathrm{CH})$; and (B) from the other 25 countries (22 other EU MS, plus Iceland and Norway). Comparisons were made with the contributions of (1) the other four target MS, or for the other 25 countries, all five; (2) the USA; (3) the other 25 countries; and (4) the rest of the world. These comparisons were designed to show the contribution of the UK in context on a fractional count basis (adding up to 100\%). For example, a paper with two French addresses and one German one would be counted 1 each for FR and DE on integer counting, but 0.67 and 0.33 respectively on fractional counting. However, there are some papers that do not have addresses, meaning that the number of classified papers in the results section on the Tables does not correspond to the overall number of cited research papers (left-hand column "Papers", on "Total") as these were excluded from any analysis.

\section{Comparator analysis}

The UK percentage presence in the respiratory conditions research was determined for the world excluding each of the five target EU MSs and the other $25 \mathrm{EU}$ MSs. These percentages were then compared with the UK contributions to papers cited by the RESPI clinical guidelines to these MSs. All data were calculated on a fractional count as this gives a better understanding to the actual contribution of the UK to other countries' guidelines.

\section{Results}

The results presented are for the UK contribution to CPGs published by the five target Member States and by the other Member

\begin{tabular}{|c|c|c|c|c|c|}
\hline EU MS & Organisations & RESPI CPGs & AST CPGs & COP CPGs & $\begin{array}{l}\text { Cited references in } \\
\text { CPGs }\end{array}$ \\
\hline $\mathrm{DE}$ & $\begin{array}{l}\text { Arbeitsgemeinschaft der Wissenschaftlichen Medizinischen Fachgesellschaften (AWMF) } \\
\text { [Association of Scientific Medical Societies] }\end{array}$ & 4 & 1 & 3 & 844 \\
\hline FR & Haute Authorité de Santé (HAS) [High Authority of Health] & 2 & 2 & $0^{*}$ & 215 \\
\hline NL & Centraal Begeleidings Orgaan (CBO) [Central Counseling Agency] & 3 & 0 & 3 & 501 \\
\hline SE & Socialstyrelsen [The National Board of Health and Welfare] & 1 & 1 & 0 & 145 \\
\hline $\mathrm{CH}$ & Swiss Respiratory Society & 1 & 0 & 1 & 109 \\
\hline EUR5 & Germany, France, the Netherlands, Sweden, Switzerland & 11 & 4 & 7 & 1814 \\
\hline EUR13 & $\begin{array}{l}\text { Austria, Belgium, Bulgaria, Croatia, Czech Republic, Denmark, Estonia, Finland, Hungary, } \\
\text { Lithuania, Portugal, Romania, Spain }\end{array}$ & 41 & 22 & 19 & 4273 \\
\hline Total & 18 Member States & 52 & 26 & 26 & 6087 \\
\hline \multicolumn{6}{|c|}{$\begin{array}{l}\text { CPGs: Clinical Practice Guidelines; RESPI: Respiratory Conditions; AST: Asthma; COPD: Chronic Obstructive Pulmonary Disease; DE: Germany; FR: France; NL: The } \\
\text { Netherlands; SE: Sweden; CH: Switzerland; EUR5: DE, FR, NL, SE CH } \\
\text { "For FR although there was a CPG on COPD, this was not included in the analysis as the cited references were a few ( } n=20) \text { and although included other clinical } \\
\text { practice guidelines from other countries including the UK, it would not reach any meaningful results among the hundreds of analysed references. }\end{array}$} \\
\hline
\end{tabular}

Table 1: The organisations involved in the production of respiratory clinical practice guidelines on asthma and chronic obstructive pulmonary disease in each of the five target EU MSs, the other 13 European Member States and their cited references.

\begin{tabular}{|c|c|c|c|}
\hline NCD & Cause & Application Code & EUR31 (average \%) \\
\hline RESPI & Chronic obstructive pulmonary disease & COP \\
\hline RESPI & Asthma & 2.95 \\
\hline
\end{tabular}

Table 2: The top $1 \%$ of the EUR31 countries' DALYs (average) in chronic respiratory conditions. 
Citation: Pallari E (2017) The Value of UK Respiratory Conditions Research on Clinical Practice Guidelines from Other European Member States . J Pulm Respir Med 7: 434. doi: 10.4172/2161-105X.1000434

Page 3 of 5

States as a group. This was done for both respiratory conditions' clinical guidelines collectively and for the two respiratory conditions, asthma and chronic obstructive pulmonary disease (COPD) separately. The results presented are on a fractional count basis. There were 52 clinical guidelines on two respiratory conditions, asthma and COPD, in 18 European countries with a total number of 6,087 cited references, as shown in Table 1.

\section{Overall respiratory conditions}

On overall respiratory conditions, the other nine EU MSs are Austria, Belgium, Bulgaria, Croatia, Czech Republic, Denmark, Estonia, Finland, Hungary, Lithuania, Portugal, Romania and Spain. Table 3 shows raw values and UK contribution on a fractional count basis. The UK contribution to other EU Member States clinical guidelines shows that about one in six research papers is cited in a country's own clinical guidelines portfolio as shown in Tables 3-5. The UK contribution is in terms of a percentage presence of different countries (Tables 4 and 5). This is higher than the five target EU MSs collectively (column other 4) and higher than the rest of the EU MSs' contribution. However, the European contribution to UK guidelines was less than that of the USA or the Rest of the World (RoW). The presence of different countries as a percentage of foreign collaboration, shows that UK is higher than the other four EU MSs, but almost equal to the rest of Europe collectively, and less than the rest of the world or the US, shown in Table 4.

\begin{tabular}{|c|c|c|c|c|c|c|c|}
\hline EU MSs & Papers & Own CU & Other 4 & UK & US & EUR 25 & RoW \\
\hline DE & 844 & 30.1 & 109.6 & 144.3 & 238.5 & 130.7 & 166.9 \\
\hline FR & 215 & 12.5 & 21.4 & 32.9 & 46.4 & 37.7 & 62.1 \\
\hline NL & 501 & 55.6 & 38.8 & 80.5 & 148.1 & 78.9 & 81.1 \\
\hline SE & 145 & 6.89 & 11.2 & 15.9 & 35.2 & 28.1 & 45.6 \\
\hline CH & 109 & 8.79 & 16.4 & 14.2 & 33.1 & 18 & 17.5 \\
\hline EUR9 & 4273 & 481.9 & 582.7 & 750.4 & 1170.3 & 255.8 & 927.8 \\
\hline Total & 6087 & 595.78 & 780.1 & 1038.2 & 1671.6 & 549.2 & 1301 \\
\hline \multicolumn{7}{|c|}{ CU: Country contribution } \\
\hline
\end{tabular}

Table 3: Contributions (fractional counts of papers) from target MS, the UK the USA, the other 25 European countries and the Rest of the World to clinical guidelines from the target MS and the other nine EU MS on respiratory conditions overall.

\begin{tabular}{|c|c|c|c|c|c|c|}
\hline EU MSs & Own CU & Other 4 & UK & US & EUR 25 & RoW \\
\hline DE & 3.6 & 13.0 & 17.1 & 28.3 & 15.5 & 19.8 \\
\hline FR & 5.8 & 10.0 & 15.3 & 21.6 & 17.5 & 28.9 \\
\hline NL & 11.1 & 7.7 & 16.1 & 29.6 & 15.7 & 16.2 \\
\hline SE & 4.8 & 7.7 & 11.0 & 24.3 & 19.4 & 31.4 \\
\hline CH & 8.1 & 15.0 & 13.0 & 30.4 & 16.5 & 16.1 \\
\hline EUR9 & 11.3 & 13.6 & 17.6 & 27.4 & 6.0 & 21.7 \\
\hline
\end{tabular}

Table 4: Contributions (percentages of papers, fractional counts) from target MS the UK, the USA, the other 25 European countries and the Rest of the World to clinical guidelines from the target MS and the other nine EU MS on respiratory conditions overall.

\begin{tabular}{|c|c|c|c|c|c|c|}
\hline EU MSs & Other 4 & UK & US & EUR 25 & RoW & None \\
\hline DE & 13.5 & 17.7 & 29.3 & 16.1 & 20.5 & 2.9 \\
\hline FR & 10.6 & 16.2 & 22.9 & 18.6 & 30.7 & 1.0 \\
\hline NL & 8.7 & 18.1 & 33.3 & 17.7 & 18.2 & 4.0 \\
\hline SE & 8.1 & 11.5 & 25.5 & 20.3 & 33.0 & 1.5 \\
\hline CH & 16.4 & 14.2 & 33.0 & 18.0 & 17.5 & 1.0 \\
\hline EUR9 & 15.4 & 19.8 & 30.9 & 6.7 & 24.5 & 2.7 \\
\hline
\end{tabular}

Table 5: Contributions (percentages of foreign contributions to papers, fractional counts) from target MS, the UK, the USA, the other 25 European countries and the Rest of the World to clinical guidelines from the target MS and the other nine EU MS on respiratory conditions overall.

\section{Asthma}

On asthma, the other six EU MSs are Croatia, Czech Republic, Finland, Hungary, Lithuania, Portugal, Romania and Spain. Table 6 shows raw values and UK contribution on a fractional count basis. The UK contribution is in terms of a percentage presence of different countries (Tables 7 and 8). The UK contribution to other EU Member States clinical guidelines varies between $11-19 \%$ of research papers cited in a country's own clinical guidelines portfolio as shown in Tables 7 and 8. For asthma, this is higher than own country's contribution, and higher than the five target EU MSs collectively (column other 4) but less than the rest of the EU MSs' contribution (Table 7). The UK contribution though, is less than the Rest of the World or the United States and is also comparable on foreign contribution too (Table 8).

\section{COPD}

On COPD, the other nine EU MSs are Austria, Belgium, Bulgaria, Czech Republic, Denmark, Finland, Hungary, Lithuania and Spain. Table 9 shows the raw values and UK contribution on a fractional count basis. The UK contribution is in terms of a percentage presence of different countries (Tables 10 and 11). The UK contribution to other EU Member States clinical guidelines varies between 11-19\% of research papers cited in a country's own clinical guidelines portfolio as shown in Tables 10 and 11. For asthma, this is higher than own country's contribution or than the five target EU MSs collectively (column other 4) but less than the rest of the EU MSs' contribution (Table 10). The UK

Asthma

\begin{tabular}{|c|c|c|c|c|c|c|c|}
\hline EU MSs & Papers & Own CU & Other 4 & UK & US & EUR 25 & RoW \\
\hline DE & 388 & 15.7 & 50.4 & 65.6 & 101.2 & 55.7 & 89.4 \\
\hline FR & 215 & 12.5 & 21.4 & 32.9 & 46.4 & 37.7 & 62 \\
\hline NL & 0 & 0 & 0 & 0 & 0 & 0 & 0 \\
\hline SE & 145 & 6.89 & 11.2 & 15.9 & 35.2 & 28.1 & 45.6 \\
\hline CH & 0 & 0 & 0 & 0 & 0 & 0 & 0 \\
\hline EUR6 & 1898 & 108.1 & 234.2 & 356.3 & 510.4 & 180.8 & 456.9 \\
\hline Total & 2646 & 143.19 & 317.2 & 470.7 & 693.2 & 302.3 & 653.9 \\
\hline
\end{tabular}

Table 6: Contributions (fractional counts of papers) from target MS, the UK the USA, the other 25 European countries and the Rest of the World to clinical guidelines from the target MS and the other six EU MS on asthma.

\begin{tabular}{|c|c|c|c|c|c|c|}
\hline EU MSs & Own CU & Other 4 & UK & US & EUR 25 & RoW \\
\hline DE & 4.0 & 13.0 & 16.9 & 26.1 & 14.4 & 23.0 \\
\hline FR & 5.8 & 10.0 & 15.3 & 21.6 & 17.5 & 28.8 \\
\hline NL & 0 & 0 & 0 & 0 & 0 & 0 \\
\hline SE & 4.8 & 7.7 & 11.0 & 24.3 & 19.4 & 31.4 \\
\hline CH & 0 & 0 & 0 & 0 & 0 & 0 \\
\hline EUR6 & 5.7 & 12.3 & 18.8 & 26.9 & 9.5 & 24.1 \\
\hline
\end{tabular}

Table 7: Contributions (percentages of papers, fractional counts) from target MS the UK, the USA, the other 25 European countries and the Rest of the World to clinical guidelines from the target MS and the other six EU MS on asthma.

\begin{tabular}{|c|c|c|c|c|c|}
\hline EU MSs & Other 4 & UK & US & EUR 25 & RoW \\
\hline DE & 13.5 & 17.6 & 27.2 & 15.0 & 24.0 \\
\hline FR & 10.6 & 16.2 & 22.9 & 18.6 & 30.6 \\
\hline NL & 0.0 & 0.0 & 0.0 & 0.0 & 0.0 \\
\hline SE & 8.1 & 11.5 & 25.5 & 20.3 & 33.0 \\
\hline CH & 0.0 & 0.0 & 0.0 & 0.0 & 0.0 \\
\hline EUR6 & 13.1 & 19.9 & 28.5 & 10.1 & 25.5 \\
\hline
\end{tabular}

Table 8: Contributions (percentages of foreign contributions to papers, fractional counts) from target MS, the UK, the USA, the other 25 European countries and the Rest of the World to clinical guidelines from the target MS and the other six EU MS on asthma. 


\section{COPD}

\begin{tabular}{|c|c|c|c|c|c|c|c|}
\hline EU MSs & Papers & Own CU & Other 4 & UK & US & EUR 25 & RoW \\
\hline DE & 456 & 14.4 & 59.2 & 78.7 & 137.3 & 75 & 77.4 \\
\hline FR & 0 & 0 & 0 & 0 & 0 & 0 & 0 \\
\hline NL & 501 & 55.6 & 38.8 & 80.5 & 148.1 & 78.9 & 81.1 \\
\hline SE & 0 & 0 & 0 & 0 & 0 & 0 & 0 \\
\hline CH & 109 & 8.79 & 16.4 & 14.2 & 33.1 & 18 & 17.5 \\
\hline EUR9 & 2366 & 291.3 & 346.8 & 392.7 & 655.6 & 155.8 & 469.7 \\
\hline Total & 3432 & 370.09 & 461.2 & 566.1 & 974.1 & 327.7 & 645.7 \\
\hline
\end{tabular}

Table 9: Contributions (fractional counts of papers) from target MS, the UK the USA, the other 25 European countries and the Rest of the World to clinical guidelines from the target MS and the other nine EU MS on chronic obstructive pulmonary disease.

\begin{tabular}{|c|c|c|c|c|c|c|}
\hline EU MSs & Own CU & Other 4 & UK & US & EUR 25 & RoW \\
\hline DE & 3.2 & 13.0 & 17.3 & 30.1 & 16.4 & 17.0 \\
\hline FR & 0 & 0 & 0 & 0 & 0 & 0 \\
\hline NL & 11.1 & 7.7 & 16.1 & 29.6 & 15.7 & 16.2 \\
\hline SE & 0 & 0 & 0 & 0 & 0 & 0 \\
\hline CH & 8.1 & 15.0 & 13.0 & 30.4 & 16.5 & 16.1 \\
\hline EUR9 & 12.3 & 14.7 & 16.6 & 27.7 & 6.6 & 19.9 \\
\hline
\end{tabular}

Table 10: Contributions (percentages of papers, fractional counts) from target MS, the UK, the USA, the other 25 European countries and the Rest of the World to clinical guidelines from the target MS and the other nine EU MS on chronic obstructive pulmonary disease.

\begin{tabular}{|c|c|c|c|c|c|}
\hline EU MSs & Other 4 & UK & US & EUR 25 & RoW \\
\hline DE & 13.4 & 17.8 & 31.1 & 17.0 & 17.5 \\
\hline FR & 0.0 & 0.0 & 0.0 & 0.0 & 0.0 \\
\hline NL & 8.7 & 18.1 & 33.3 & 17.7 & 18.2 \\
\hline SE & 0.0 & 0.0 & 0.0 & 0.0 & 0.0 \\
\hline CH & 16.4 & 14.2 & 33.0 & 18.0 & 17.5 \\
\hline EUR9 & 16.7 & 18.9 & 31.6 & 7.5 & 22.6 \\
\hline
\end{tabular}

Table 11: Contributions (percentages of foreign contributions to papers, fractional counts) from target MS, the UK, the USA, the other 25 European countries and the Rest of the World to clinical guidelines from the target MS and the other nine EU MS on chronic obstructive pulmonary disease.

\begin{tabular}{|c|c|c|c|}
\hline EU MSs & Refs on CPGs, UK \% & Research, UK \% & Ratio \\
\hline DE & 17.73 & 12.29 & 1.44 \\
\hline FR & 16.25 & 12.36 & 1.31 \\
\hline NL & 18.07 & 12.19 & 1.48 \\
\hline SE & 11.51 & 11.98 & 0.96 \\
\hline CH & 14.17 & 11.79 & 1.2 \\
\hline EUR25 & 19.79 & 14.59 & 1.36 \\
\hline
\end{tabular}

Table 12: UK presence: Percentages of its contributions to references on CPGs and percentages of respiratory conditions' research excluding papers from the different EU MS, and the ratio between them.

\begin{tabular}{|c|c|c|c|c|c|c|c|c|c|}
\hline \multirow{2}{*}{$\begin{array}{c}\text { CD } \\
\text { Period }\end{array}$} & \multicolumn{3}{|c|}{ RESPI } & \multicolumn{3}{|c|}{ AST } & \multicolumn{3}{|c|}{ COP } \\
\hline & Total & UK, frac & UK, \% & Total & UK, frac & UK, \% & Total & UK, frac & UK, \% \\
\hline$<1990$ & 399 & 81.5 & 20.43 & 87 & 29.4 & 33.79 & 312 & 52.2 & 16.73 \\
\hline 1990-1994 & 432 & 76.2 & 17.64 & 166 & 33.5 & 20.18 & 265 & 42.7 & 16.11 \\
\hline 1995-1999 & 1121 & 152.5 & 13.6 & 457 & 76.4 & 16.72 & 663 & 75.1 & 11.33 \\
\hline $2000-2004$ & 1746 & 338.8 & 19.4 & 843 & 151.4 & 17.96 & 901 & 187.5 & 20.81 \\
\hline $2005-2009$ & 1588 & 257.9 & 16.24 & 791 & 137.7 & 17.41 & 788 & 119.8 & 15.2 \\
\hline $2010-2014$ & 801 & 130.3 & 16.27 & 299 & 41.4 & 13.85 & 502 & 88.9 & 17.71 \\
\hline
\end{tabular}

Table 13: Numbers of clinical guidelines' cited papers (N) from the five target EU MS and the 15 others in five-year periods beginning before 1990 s until 2014 , and the UK participation on fractional counts and percentage contribution.

contribution though, is less than the Rest of the World or the United States and is also comparable on foreign contribution too (Table 11).

\section{Comparison of UK's presence on CPG references with its presence in respiratory conditions research}

The UK percentage contribution to the evidence base of clinical guidelines from five target EU MS (Germany, France, Netherland, Sweden and Switzerland), and other continental European countries, is shown on Table 12 for all respiratory conditions, asthma and COPD. The papers from 2002-2013 were compared on a fractional output basis with the papers in the Web of Science, compared with its presence in research in 2002-2013, all-fractional counts.

\section{Variation of UK participation with time}

Some of the references on the clinical guidelines go back before the start of the study year 2002. Therefore, to examine the variation of UK participation with time and assess the impact on research citation in the CPGs, the UK cited research papers were classified into six broad five-year periods: before 1990, 1990-1994, 1995-1999, 2000-2004, 20052009, 2010-2014 for the three NCD fields as shown in Table 13. The participation of the UK in the CPGs decreased slightly with time for all respiratory conditions. For asthma, particularly there has been a great decrease of UK research in the European clinical guidelines evidence base. For COPD, there was a drop between 1995-1999 but then a sharp increase and during the last five-year period an increase.

\section{Discussions}

This study was set to examine the research landscape of Europe in the form of UK influence on clinical guidelines' development. There is a substantial percentage contribution from UK institutions underpinning clinical practice guidelines' recommendations. This contribution of UK research to other EU MS's research is even greater on CPGs than research paper output as indicated by the ratios in Table 12. Perhaps this difference could indicate the important relevance of UK research as applied in the form of recommendations than other conventional research output measures. A limitation of this study though is the lack of examination of the contribution of European Member States' research on the UK clinical practice guidelines. A future piece of research work could be the examination of the UK CPGs for the contribution from individual European countries in the underpinned research evidence-base and the establishment of further impact on formed recommendations. This in particular could be of interest, as from 2010-2014 there has been a decrease on the UK contribution to asthma research and slightly more on COPD from the previous five years. This may indicate the need to strike a balance between research attention between asthma and COPD and even the consideration of other conditions like emphysema, bronchitis or cystic Fibrosis (which were not included in this study). The clinical importance of such a shift in the trend of the cited research evidence-base for clinical practice may be due to a potential shift towards new or experimental treatments 
tackling COPD. Although this cannot be inferred in this study, a previous study demonstrated that the citation of asthma research in European CPGs was more clinical than COPD [7] while research reported on newspaper stories was more basic than that of COPD [8]. Also, the latter study [8] demonstrated a heavily reporting research on asthma than COPD despite the reverse image presented by the disease burden afflicted by the European population. However, with limited funding, such research practices may even lead to further variation between countries' CPG focus and even greater disparities between the different respiratory conditions.

\section{Conclusions}

This study assessed the UK contribution to the evidence-base of other European countries' respiratory conditions' clinical practice guidelines. The UK contribution to the evidence-base of respiratory clinical guidelines was about a sixth for the five key European Member States and for the rest of the EU. The study showed the importance of UK medical research to healthcare in other European MS based on evidence-based recommendations. The UK participation in the clinical guidelines' references decreased slightly over time for asthma but increased for COPD. The extent of this impact on clinical practice though remains unknown. It would be of interest to see how such studies contributing to evidence-based recommendations are influencing clinical practice or patient outcomes. Assessing the adherence or implementation of such evidence-based recommendations from these various European respiratory conditions guidelines and comparing their impact in practice can have further implications for research, finding and collaboration. Potential implications of the current findings are that UK medical research is important for healthcare in other EU MS, so they have an interest in ensuring that it is not adversely affected by the UK's departure from the EU.

\section{Funding}

The research was funded by the European Community's Seventh Framework Programme, Grant/Award Number: EC/FP7/602536. EP is currently funded by The Urology Foundation (TUF) and also supported from funding provided by the National Institute for Health Research (NIHR) and The Collaboration for Leadership in Applied Health Research and Care (CLAHRC South London).

\section{Acknowledgments}

EP would like to thank Prof Tony W. Fox from the KCL Faculty of Pharmaceutical Medicine for his comments on the manuscript. The author would like to thank Dr Philip Roe for the development of the VBA programs used for the analysis of the cited papers. Also, the author would like to thank Dr Grant Lewison and Ms Mursheda Begum from the Division of Cancer Studies for the provision of the research output data. EP trained and coordinated data collection from the following individuals on the clinical guidelines and would like to thank: for Austria, Germany and Switzerland: Natalia Kelsch, Anne Spranger, Victor Stephan and Tobias Schumacher from the Technische Universität Berlin, Berlin, Germany; for Belgium: Ann-Sophie de Mol and Gabrielle Emanuel King's College London
(KCL); for Bulgaria: Eva Nacheva, Christina Tencheva from KCL; for Croatia: Ria Ivandic from KCL; for Czech Republic and Poland: Kasia Zemanek from KCL; for Denmark: Maria Dahl and Maria Emilsson from KCL; for Estonia: Argo Soon from the Estonian Research Council, Tartu; for Finland: Laura Mantovani from KCL; for France: Anshoo Lumba and Karen Bringham from the Université Paris Est Créteil in Paris; for Hungary: Csajbok Edit, from the Semmelweis University in Budapest; for Italy: Ludovica Borsoi from the Universita Commerciale Luigi Bocconi in Milan; for Lithuania and Latvia: Marleen Saidla and Ingrid Jaselskyte from the Estonian Research Council in Tartu; for the Netherlands: Ann-Sophie de Mol from KCL; for Portugal: Diana Gosálvez-Prados, Elisabeth María Ilidio-Paulo, Camila Higueras-Callejón and José Carlos Ruiz-Jiménez from the Escuela Andaluza de Salud Publica in Granada; for Romania: Maria-Cristina Juverdeanu from KCL; for Spain: Diana Gosálvez-Prados and Elena Salamanca-Fernández from the Escuela Andaluza de Salud Publica in Granada; for Sweden: Gustaf Nelhans from the Sahlgrenska Göteborg.

\section{References}

1. Straus SE, Sackett DL (1998) Using research findings in clinical practice. BMJ 317: 339.

2. Haines A, Donald A (1998) Making better use of research findings. BMJ 317: 72.

3. Haynes B, Haines A (1998) Barriers and bridges to evidence based clinical practice. BMJ 317: 273.

4. Grimshaw J, Freemantle N, Wallace S, Russell I, Hurwitz B, et al. (1995) Developing and implementing clinical practice guidelines. Qual Health care 4: 55 .

5. Bazian Ltd. (2005) Do evidence-based guidelines improve the quality of care? Evidence-Based Healthcare and Public Health 9: 270-275.

6. Grimshaw JM, Russell IT (1993) Effect of clinical guidelines on medical practice: a systematic review of rigorous evaluations. The Lancet 342: 1317-1322.

7. Begum M, Lewison G, Wright JS, Pallari E, Sullivan R (2016) European noncommunicable respiratory disease research, 2002-13: Bibliometric study of outputs and funding. PLoS One 11: e0154197.

8. Pallari E, Lewison G, Sullivan R (2017) How is chronic non-communicable respiratory conditions research reported in European newspapers? An impact assessment for policy. Clin Respir J 11: 657-665.

9. European Lung White Book.

10. The UK and EU research funding

11. Legido-Quigley H, Panteli D, Car J, McKee M, Busse R (2013) Clinical guidelines for chronic conditions in the European Union. European Observatory on Health Systems and Policies.

12. https://www.has-sante.fr/portail/jcms/r_1455134/en/about-has

13. http://www.awmf.org/en/clinical-practice-guidelines/awmf-imwi.html

14. AWMF (2010) National Programme for Disease Management Guidelines.

15. Richtlijnen database guidelines

16. http://www.socialstyrelsen.se/riktlinjer/nationellariktlinjer

17. Russi EW, Karrer W, Brutsche M, Eich C, Fitting JW, et al. (2013) Diagnosis and management of chronic obstructive pulmonary disease: The Swiss guidelines. Respiration 85: 160-174 